\title{
Extending NGOMSL model for human-humanoid robot interaction in the soccer robotics domain
}

\author{
Rajesh Elara Mohan*, Carlos Antonio Acosta Calderon, Changjiu Zhou* and Pik Kong Yue \\ Advanced Robotics and Intelligent Control Centre, School of Electrical and Electronics Engineering, Singapore Polytechnic, \\ 500 Dover Road, Singapore
}

(Received 26 August 2008; final version received 10 December 2008)

\begin{abstract}
In the field of human-computer interaction, the Natural Goals, Operators, Methods, and Selection rules Language (NGOMSL) model is one of the most popular methods for modelling knowledge and cognitive processes for rapid usability evaluation. The NGOMSL model is a description of the knowledge that a user must possess to operate the system represented as elementary actions for effective usability evaluations. In the last few years, mobile robots have been exhibiting a stronger presence in commercial markets and very little work has been done with NGOMSL modelling for usability evaluations in the human-robot interaction discipline. This paper focuses on extending the NGOMSL model for usability evaluation of human-humanoid robot interaction in the soccer robotics domain. The NGOMSL modelled human-humanoid interaction design of Robo-Erectus Junior was evaluated and the results of the experiments showed that the interaction design was able to find faults in an average time of $23.84 \mathrm{~s}$. Also, the interaction design was able to detect the fault within the $60 \mathrm{~s}$ in $100 \%$ of the cases. The Evaluated Interaction design was adopted by our Robo-Erectus Junior version of humanoid robots in the RoboCup 2007 humanoid soccer league.
\end{abstract}

Keywords: NGOMSL model; humanoid robots; human-humanoid robot interaction; usability evaluation

\section{Introduction}

Growing popularity and increasingly viable application domains have contributed to the greater presence of robots in the commercial marketplace. Among various robotic platforms, humanoid robotic research especially has seen a rapid growth in the recent years due to the ability of humanoid robots to behave and interact like humans. Humanoid robots might provide day-to-day support in the home and the workplace, doing laundry or dishes, assisting in the care of the elderly, or acting as a caretaker for individuals within a home or institution (Metev and Veiko 2006). Many of these tasks will involve close interaction between the robot and the people it serves. Researchers have studied humanoid robot development, including control, emotional expressiveness, human-robot teaming, humanhumanoid robot interaction and perception (Okuno et al. 2002; Kanda et al. 2003; Edsinger and Kemp 2007). However, there have been fewer studies on evaluation models for human-humanoid robot interaction.

The Natural Goals, Operators, Methods, and Selection rules Language (NGOMSL) model is one of the popular methods for representing the procedural knowledge required to operate a system in terms of the user goals, basic actions or operators, methods, which are sequences of operators that will accomplish goals, and selection rules, which determine which method to apply to accomplish a goal (Kieras 2002). A NGOMSL model involves decomposition of a single high-level goal into a sequence of unit tasks, all of which can be further decomposed down to the level of basic operators. The NGOMSL model has served as the basis for much of the cognitive modelling research in the human computer interaction field. NGOMSL models can be classified as predictive, descriptive and prescriptive. A NGOMSL model is predictive because it can be used to predict the time needed by a user to perform the tasks under analysis, as long as the developer can come up with time estimates for the operators involved in each model. A NGOMSL model is descriptive in the sense that it is a representation of the way a user performs tasks on a system. The methods, subgoals and selection rules provide the designer with a description of the process, rather than simply a time estimate. A NGOMSL model can also be considered prescriptive as it can be used to teach new users how to achieve the goal (Hochestein 2002). Very little work has been done with NGOMSL modelling for usability evaluations of human-humanoid robot interaction in the soccer robotics domain. Scholtz, Drury and Kieras adopted NGOMSL for modelling human-robot interaction but did not explore specific issues pertaining to humanoid robots or the soccer robotics domain (Drury et al. 2007).

RoboCup is an international joint research initiative to foster artificial intelligence, robotics and related research.

*Corresponding author. Email: ZhouCJ@sp.edu.sg, MohanRajesh@sp.edu.sg 
The ultimate goal of the RoboCup initiative is to develop a team of fully autonomous humanoid robots that can win against the human world champion team in soccer. Our Robo-Erectus version of robots has been participating in the humanoid soccer league of RoboCup since 2002, collecting several awards since then. Various technologies are incorporated into a soccer robot including, multi-robot cooperation, human-humanoid robot interaction, sensor fusion, artificial intelligence, real-time processing, navigation and localization. Every league has its own requirements on the robot hardware and software systems. Humanoid 2 versus 2 soccer matches are played with two teams of two robots in autonomous mode on a $4.5 \mathrm{~m} \times 3 \mathrm{~m}$ field. Each robot must have a human-like body plan with two legs, two arms, and one head, which are attached to a trunk and fit into a cylinder of diameter $0.55 H$, where $H$ is the height of the humanoid robot. During the soccer match, due to the hardware and software complexity of a humanoid robot operating in autonomous mode system failures occur. The corresponding team requests stoppage of game with a timeout period of $120 \mathrm{~s}$ to find fault and service their robot and the game starts after the elapse of $120 \mathrm{~s}$ (Menegatti et al. 2007). Therefore, a humanoid 2 versus 2 soccer match requires human-humanoid robot interaction design to be able to detect the system fault with the robot as early as possible. Experimental results have shown that the interaction design must be able to find the fault with the robot in at least $60 \mathrm{~s}$ (half the timeout period) so as to enable the user to fix the fault within the timeout period. Delay in finding faults during the timeout period might provide an edge for the opponents team as they can continue playing the game after the elapse of $120 \mathrm{~s}$.

This paper focuses on extending the NGOMSL model for usability evaluations in human-humanoid robot interaction in the soccer domain. The NGOMSL modelled humanhumanoid interaction design of Robo-Erectus Junior was evaluated and the results of the experiments showed that the interaction design was able to find faults in an average time of $23.84 \mathrm{~s}$. Also, the interaction design was able to detect the fault within $60 \mathrm{~s}$ in $100 \%$ of the cases. Our Robo-Erectus Junior humanoid robot was equipped with the NGOMSL modelled interaction design in the RoboCup 2007 humanoid soccer league. The rest of the paper is organized as follows: Section 2 discusses the NGOMSL model and its application in the human-humanoid robot interaction domain, while Section 3 provides a brief introduction to our humanoid platform, Robo-Erectus Junior, and its humanhumanoid robot interaction design. Section 4 summarizes the experiments performed and the results. Finally, Section 5 provides the conclusion and future work.

\section{NGOMSL model for usability evaluation}

The NGOMSL model was developed by David Kieras in 1988. It was formulated to make the standard GOMS model
Table 1. Standard primitive descriptors of NGOMSL model.

\begin{tabular}{|c|c|c|}
\hline Operator name & Description & Time (s) \\
\hline$K$ & $\begin{array}{l}\text { Pressing a single key or } \\
\text { button }\end{array}$ & 0.35 \\
\hline$K 1$ & $\begin{array}{l}\text { Pressing shift or control } \\
\text { keys }\end{array}$ & 0.08 \\
\hline$P$ & $\begin{array}{l}\text { Pointing with a mouse or } \\
\text { other device to a target } \\
\text { on a display }\end{array}$ & 1.10 \\
\hline$P 1$ & $\begin{array}{l}\text { Clicking the mouse or } \\
\text { similar device }\end{array}$ & 0.20 \\
\hline$H$ & $\begin{array}{l}\text { Homing hands on the } \\
\text { keyboard or other } \\
\text { device }\end{array}$ & 0.40 \\
\hline$M$ & $\begin{array}{l}\text { Mentally prepare to do } \\
\text { something }\end{array}$ & 1.35 \\
\hline$R(t)$ & $\begin{array}{l}\text { System response time - } \\
\text { counted only if it } \\
\text { causes the user to wait } \\
\text { when carrying out } \\
\text { their task. }\end{array}$ & $t$ (variable) \\
\hline
\end{tabular}

simple to use and still hold its vital features. The NGOMSL model is based on cognitive complexity theory with the ability to estimate both the execution time and time taken to learn how to use the system. The NGOMSL model does not allow complicated conditional statements as it assumes that humans cannot process complex conditionals in a single cognitive step. The key components of NGOMSL are defined (Kieras 1997) in the following:

Goals: Goals are what the user wishes to achieve. It can be defined at various levels of abstraction, from very highlevel goals to low-level goals. Higher-level goals are decomposable into subgoals, and are arranged hierarchically.

Operators: Operators are the elementary perceptual, motor or cognitive actions that are used to accomplish the goals. Operators are not decomposable: they are atomic elements in the NGOMSL model. Furthermore, it is generally assumed that each operator requires a fixed amount of time for the user to execute, and that this time interval is independent of context. Table 1 shows the standard primitive operators of the NGOMSL model represented by a oneletter code and their respective standard time durations.

Methods: Methods are the procedures that describe how to accomplish goals. A method is essentially an algorithm that the user has internalised that determines the sequence of subgoals and operators necessary to achieve the desired goal. There may exist more than one method to achieve a single goal.

Selection Rule: Selection rules specify which method should be used to satisfy a given goal, based on the context. Since there may be several different ways of achieving the same goal, selection rules represent the user's knowledge of which method must be applied to achieve the desired goal. 
Selection rules generally take the form of a conditional statement.

The NGOMSL model breaks the highest-level goal into subgoals which are described using a suitable method and the lowest level methods comprises of a series of primitive operators. Both the estimated execution times and learning time can be computed from the structured NGOMSL model of the interaction design.

\subsection{NGOMSL model for HHRI in soccer domain}

In the humanoid soccer domain, one of the key requirements set for the interaction design is to detect the hardware/software fault in the robot within $60 \mathrm{~s}$ of the timeout period. One of the issues with using the GOMSL model for human-humanoid robot interaction in the soccer domain is modelling the amount of time required for user's mental preparation while using the interaction system, $M$. In the human-computer-interaction domain, the user's mental preparation time period is standardized to $1.35 \mathrm{~s}$ (Preece et al. 2007) but with the autonomous, complex and dynamic nature of the humanoid soccer robot system the user's mental preparation time period, would increase. The user's mental preparation time period, $M$ now has to encompass parameters including the complexity of the system, its autonomous nature, sensor and actuator status display quality. Repeated RoboCup humanoid soccer simulated experiments performed showed that for humanoid soccer robotic applications, the user's mental preparation time period $M$ was found to be $3.55 \mathrm{~s}$.

The standard primitive does not include the time period to manipulate the humanoid robot for locomotion fault testing including soccer skills like walking, diving, kicking, recovery and so on. Therefore, we define a Locomotion operator $L$ to describe the time period for manipulating the soccer humanoid robot to perform a soccer skill. Repeated experiments showed that for humanoid soccer robotic applications, the time period for the Locomotion operator, $L$ was $4.65 \mathrm{~s}$. Manipulating the robot to perform soccer skills can help detect the hardware and software faults associated with the locomotion. Also, the standard primitive does not include the time period to run test routines to detect software faults in the artificial intelligence algorithm of the humanoid soccer robot. Therefore, we define an AI Test operator AI to describe the time period for running a test routine to detect software faults in the artificial intelligence algorithm of the humanoid soccer robot. Repeated experiments showed that for humanoid soccer robotic applications, the time period for AI Test operator was $3.75 \mathrm{~s}$. The NGOMSL model allows designer to add new operator as and when required to accommodate the increasing complexity of the robot task.

\section{Robo-Erectus Junior-A humanoid}

This Section describes the Robo-Erectus Junior humanoid robot and its human-humanoid interaction systems.

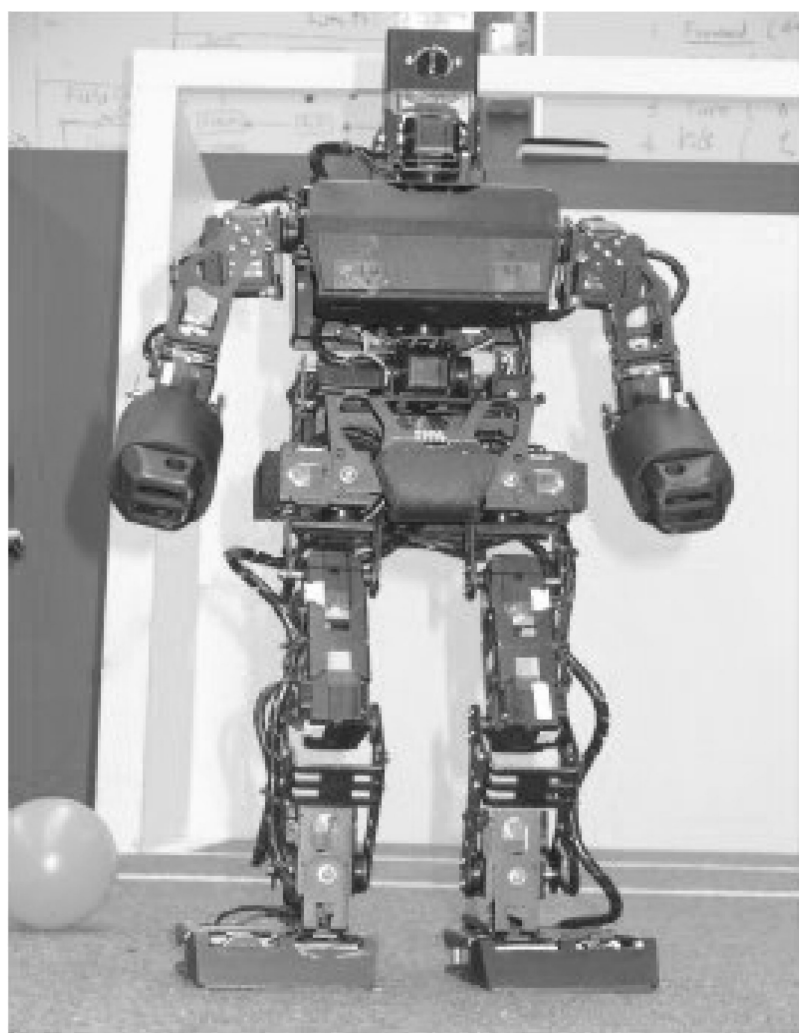

Figure 1. Robo-Erectus Junior, the Latest Generation of the Family Robo-Erectus.

The Robo-Erectus project is developed in the Advanced Robotics and Intelligent Control Centre of Singapore Polytechnic. Robo-Erectus Junior is one of the leading soccer playing humanoid robots in the RoboCup Humanoid League. Robo-Erectus Junior got the second place in the Humanoid Walk competition at the RoboCup 2002 and won the first place in Humanoid Free Performance competition at the RoboCup 2003. In 2004, Robo-Erectus Junior won the second place in Humanoid Walk, Penalty Kick and Free Performance.

The aim of the Robo-Erectus Junior development team is to develop a low-cost humanoid platform for edutainment and soccer robotics applications. The development of Robo-Erectus Junior has gone through many stages in the design of its mechanical structure, electronic control system and gait movement control. Figure 1 shows the physical design of Robo-Erectus Junior. Robo-Erectus Junior has been designed to cope with the complexity of a 2 versus 2 soccer game. It has three processors each for vision, artificial intelligence and control. Table 2 shows the specification of the processors used in Robo-Erectus Junior. The platform is equipped with three sensors: a USB camera to capture images, a tilt sensor to detect a fall and a compass to detect their direction (Zhou and Yue 2004). The servomotors used send back the feedback data including angular positions, speed, voltage and temperature. To communicate with its 
Table 2. Processor specification of Robo-Erectus Junior.

\begin{tabular}{llll}
\hline Features & $\begin{array}{l}\text { Artificial } \\
\text { intelligence } \\
\text { processor }\end{array}$ & $\begin{array}{l}\text { Vision } \\
\text { processor }\end{array}$ & $\begin{array}{l}\text { Control } \\
\text { processor }\end{array}$ \\
\hline Processor & $\begin{array}{l}\text { Intel ARM } \\
\text { Xscale }\end{array}$ & $\begin{array}{l}\text { Intel ARM } \\
\text { Xscale }\end{array}$ & $\begin{array}{c}\text { ATMEL } \\
\text { Atmega-128 }\end{array}$ \\
Speed & $400 \mathrm{Mhz}$ & $400 \mathrm{Mhz}$ & $16 \mathrm{Mhz}$ \\
Memory & $16 \mathrm{MB}$ & $32 \mathrm{MB}$ & $4 \mathrm{~KB}$ \\
Storage & $16 \mathrm{MB}$ & $16 \mathrm{MB}$ & $132 \mathrm{~KB}$ \\
Interface & RS232, WIFI & RS232, USB & RS232, RS485 \\
\hline
\end{tabular}

teammates, Robo-Erectus Junior uses a wireless network connected to the artificial intelligence processor.

The vision processor performs recognition and tracking of objects of interest including ball, goals, field lines, goal posts, teammates and the opponents based on a blob finder based algorithm. The further processing of detected blobs, wireless communications and decision making are performed by the artificial intelligence processor which selects and implements the soccer skills (e.g. walking, ball passing, kicking, diving....) the robot is to perform. Finally, the control processor handles the low-level control of motor based on the soccer skill selected by the artificial intelligence processor. Robo-Erectus Junior was fabricated to participate in the RoboCup 2007 in the KidSize category.

Table 3 shows the physical specifications of RoboErectus Junior. It is powered by two high-current Lithiumpolymer rechargeable batteries, which are located in each foot. Each battery cell has a weight of only $110 \mathrm{~g}$ providing $12 \mathrm{~V}$, which means about 15 minutes of operation. In the RoboCup 2007 competitions, Robo-Erectus Junior participated in the 2 versus 2 Soccer Games and the Technical Challenges. It was ranked as one of the top 8 robots in RoboCup 2007 humanoid 2 versus 2 soccer games, Atlanta USA.

\subsection{Human-humanoid robot interaction system}

Figure 2 shows the human-humanoid interaction system of Robo-Erectus Junior, containing a wealth of information. A separate window shows the video streaming from the robot. On the upper left of the interface is the score board showing the current status of the soccer match below which is the simulated actual match with the field localized Robo-Erectus Junior and its teammates. On the right are

Table 3. Physical specification of Robo-Erectus Junior.

\begin{tabular}{lcccr}
\hline & \multicolumn{3}{c}{ Dimension } & \\
\cline { 2 - 4 } Weight & Height & Width & Depth & $\begin{array}{c}\text { Speed } \\
\text { walking }\end{array}$ \\
\hline $3.2 \mathrm{Kg}$ & $480 \mathrm{~mm}$ & $270 \mathrm{~mm}$ & $150 \mathrm{~mm}$ & $5 \mathrm{~m} / \mathrm{min}$ \\
\hline
\end{tabular}

the control buttons for team configuration and game status signals.

The human-humanoid robot interaction design was developed to be homogeneous so as to adopt the same interaction design for multiple robot platforms. The user can switch between team configuration controls and game status signal controls. The team configuration controls include options for assigning robot identification numbers to identify each and individual robots in their own team as well as in the opponent team, goal colours to identify own and opponent goals, and individual roles of robots like striker, goalie or hybrid. In striker mode, the robot aims to score goals by kicking the ball at the opponents' goal. In goalie mode, the robot defends its goal area by stopping the ball from the opponent. In hybrid mode, the robot plays the role of a striker or the goalie depending on the circumstances. The team configuration controls also include options for viewing the sensor and actuator status, which include display of sensor data and actuator position data.

The game status signal controls provide robot external information on the status of the match including kickoff, timeout, time off, resume, ball out, indirect kick and catch. Kickoff is a way of starting or restarting play at the start of the match, after a goal has been scored, at the start of the second half of the match or at the start of each period of extra time. Depressing the kickoff button would start the robot in the autonomous mode for a soccer game. A team may request a timeout period of $120 \mathrm{~s}$ to service their robots for software/hardware faults during a soccer match causing a stoppage of the game. Each team may take utmost one timeout per half period of the match. Depressing the timeout button would perform the test routine for the checking software faults with the artificial intelligence algorithms of the humanoid robot. Time off indicates the half time period break in the middle of the soccer game.

Depressing the time off button pauses the robot's status and depressing the resume button would start the robot from pause state into autonomous soccer playing mode. An indirect free kick is awarded to the opposing team if a player commits any offences in a manner considered by the referee to be careless, reckless or using excessive force. Depressing the indirect kick would enable the robot to start in autonomous soccer playing mode with an indirect free kick. Depressing the catch button would perform a routine check to test the goalie robot's ability to dive for catching an incoming ball. The controls under the game control signal can also be used to test the functionality of the robot hardware (sensors, onboard controllers, joints and actuators) and software (implemented algorithms).

\section{Experimental results}

We adopted the NGOMSL model for modelling the humanhumanoid robot interaction of Robo-Erectus Junior. We adopted a top-down approach from the most general user 


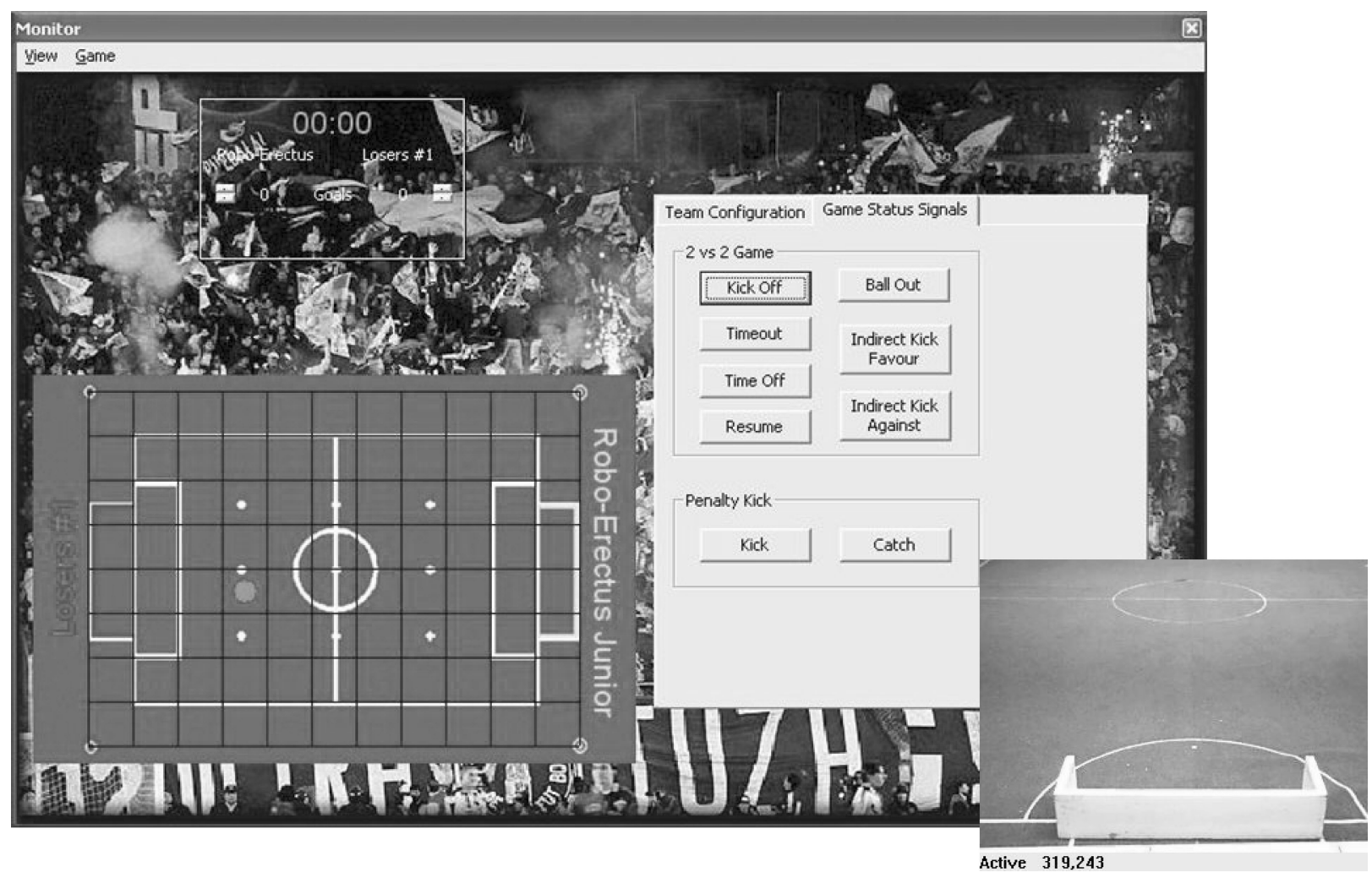

Figure 2. Human-humanoid robot interaction system of Robo-Erectus Junior.

goal to the more specific subgoals, with primitive operators at the bottom layer. All the goals in each layer are handled before going down to a lower layer. In the humanoid soccer context, we started with top-level goal of "Win a Soccer Match" as shown in Table 4. This top-level goal is then broken into subgoals, which are then broken into high-level operators. High-level operators are further broken into low-level operators, until the methods are expressed in terms of primitives. Due to space constraints, Table 5 only shows parts of the NGOMSL model for determining software/hardware faults of the soccer playing humanoid robot within the timeout period. The model shows the overall structure of the tasks to be performed. Once a fault is detected, the user calls for a timeout depressing the timeout button. Finding a fault involves running a test routine for the artificial intelligence algorithm. If the fault is not found with the algorithm, the user proceeds to perform

Table 4. NGOMSL model for top-level goal.

\begin{tabular}{ll}
\hline & Method for goal: win a soccer match \\
\hline 1 & Press game status signal button $(K)$ \\
3 & Press kick off button $(K)$ \\
4 & Accomplish goal: Score a goal \\
\hline
\end{tabular}

the locomotion test by checking the sensor and actuator data.

If the fault is not found within $60 \mathrm{~s}$ of the timeout period then the faulty robot will be replaced by another

Table 5. NGOMSL model for software/hardware fault detection.

Method for goal: find fault in robot

1 Press time off button $(K)$

2 If timeout period is greater than 60s, Accomplish goal: Replace Robot

3 Run test routine for artificial intelligence algorithm (AI)

4 If fault is not found, Accomplish goal: Perform Locomotion Test

5 Return with goal accomplished

Method for goal: Perform locomotion test

1 Make decision on the locomotion test to perform $(M)$

2 Press corresponding locomotion test button $(K)$

3 Accomplish goal: Check the sensor and actuator data

4 If fault found, return with goal accomplished

5 Go to 1

Method for goal: Check the sensor and actuator data

1 Press the team configuration button $(K)$

2 Press the display sensor \& actuator data button $(K)$

3 Wait for the system to respond $(R(t))$

4 Make decision based on the sensor \& actuator data $(M)$

5 If fault found, return with goal accomplished 


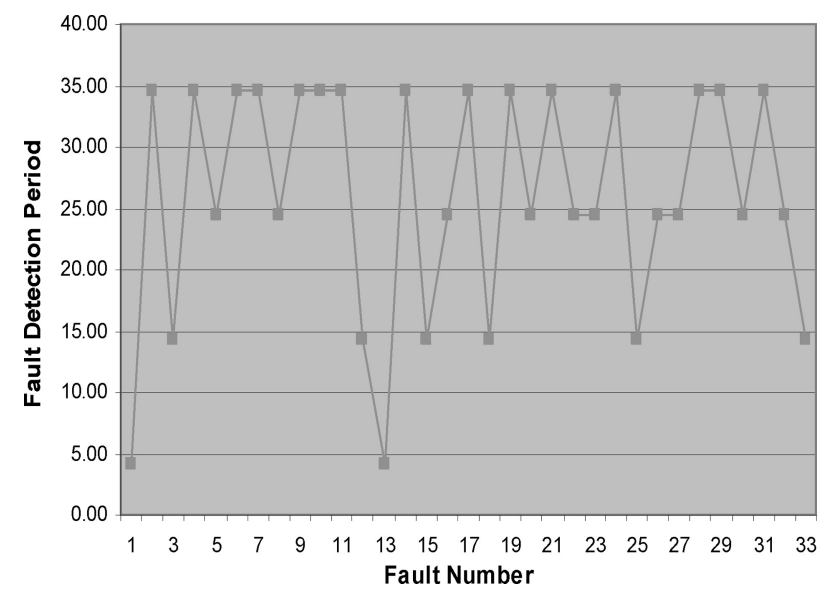

Figure 3. Fault detection period versus fault number.

backup robot. During all the experiments, the faults were found within $60 \mathrm{~s}$ of the timeout period and the replacement of robot was not required.

We conducted 20 soccer matches with the NGOMSL modelled human-humanoid robot interaction. Each match has two equal periods of 10 minutes. Players are entitled to an interval at half time. Results of the experiment showed that 33 faults occurred during 20 soccer matches. There were two faults due to the artificial intelligence algorithm and 31 faults due to locomotion related issues. In all the 33 cases, the fault was detected without having to make a replacement of the robot within the timeout period. Figure 3 shows the 33 faults and their corresponding fault detection time period with the NGOMSL model.

The average fault detection time was found to be $23.84 \mathrm{~s}$. Table 6 shows the number of software and hardware faults and their average fault detection time periods.

It was found from the experiments that the time period for detecting repeating faults tends to decrease with the number of occurrences of that particular fault. For example, the fault due to loosened screws in the knee actuator occurred four times during the soccer matches. Figure 4 shows the reduction in the time period for detection of fault due to loosened screw in the knee actuator as the number of times of occurrence of that fault increases. This decrease in fault detection time period with increasing occurrence of a particular fault is attributed to the adaptive nature of the NGOMSL model which identifies and categorizes

Table 6. Software and hardware faults and their average fault detection time periods.

\begin{tabular}{lccc}
\hline $\begin{array}{l}\text { Type of } \\
\text { Fault }\end{array}$ & $\begin{array}{c}\text { Total number of } \\
\text { soccer matches }\end{array}$ & $\begin{array}{c}\text { Number of } \\
\text { faults }\end{array}$ & $\begin{array}{c}\text { Average fault } \\
\text { detection periods (s) }\end{array}$ \\
\hline Software & 20 & 8 & $16.79 \mathrm{~s}$ \\
Hardware & 20 & 25 & $26.01 \mathrm{~s}$ \\
\hline
\end{tabular}

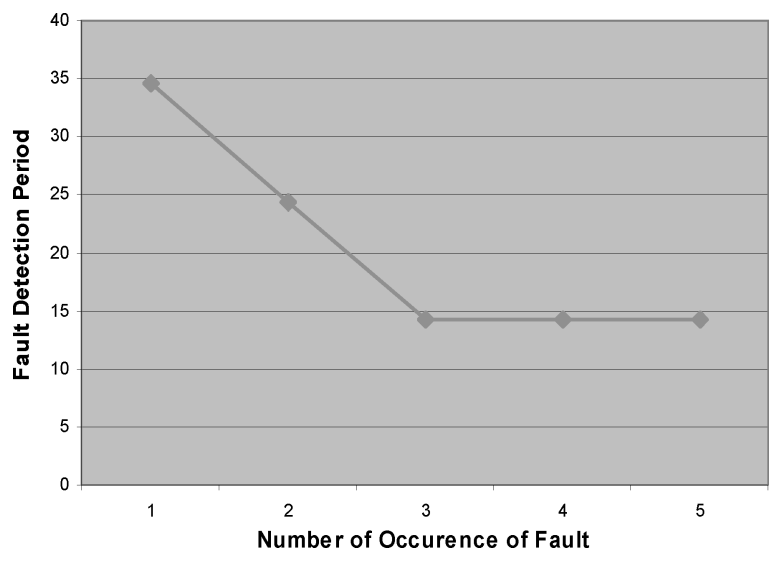

Figure 4. Fault detection period versus number of occurrence of the knee joint fault.

known faults faster as compared to unknown faults. The NGOMSL modelled human-humanoid robot interaction of Robo-Erectus Junior was adopted for RoboCup $2007 \mathrm{hu}-$ manoid soccer league at Atlanta, USA and our robot was placed as one of the top eight in the league.

\section{Conclusion}

We have presented the extension of the NGOMSL model for usability evaluation of human-humanoid robot interaction in soccer domain. The NGMOSL model was adopted for evaluating the ability of the developed interaction design to detect software/hardware faults with the robot within the timeout period in a soccer match. The average fault detection time period for the developed interaction design was found to be $23.84 \mathrm{~s}$. The NGOMSL model adopted found $100 \%$ of the faults with the robot within $60 \mathrm{~s}$ of the timeout period during the soccer matches.

The NGOMSL model also provided an estimate of the time required for performing different procedures during a soccer match helping the operator to make effective decisions. The use of NGOMSL was comparatively less costly than the user evaluation as user evaluation requires recruiting experienced evaluators, implementation of the robust version of the interaction design and prolonged testing period. This model would be further extended to analyse communication and sensor fusion related faults in multi-robot systems.

\section{References}

Drury JL, Scholtz J, Kieras D. 2007. Adapting GOMS to model human-robot interaction. Proceedings of the ACM/IEEE international conference on Human-robot interaction, Arlington, VA, USA.

Edsinger A, Kemp CC. 2007. Human-robot interaction for cooperative manipulation: Handing objects to one another. Proceedings of IEEE International Symposium on Robot and Human Interactive Communication (ROMAN), Jeju, Island, Korea. 
Hochestein L. 2002. Course Notes [online]. Available from: http://www.cs.umd.edu/class/fall2002/cmsc838s/tichi/printer/ goms.html. Access Date: 7/1/09.

Kanda T, Ishiguro H, Imai M, Ono T. 2003. Body movement analysis of human-robot interaction. Proceedings of International Joint Conferences on Artificial Intelligence (IJCAI), Acapulco, Mexico.

Kieras D. 2002. Course Notes [online]. Available from: http://www.eecs.umich.edu/ kieras/goms.html

Kieras, D. 1997. A guide to GOMS model usability evaluation using NGOMSL. In Helander M, Landauer T, and Prabhu P (Eds.), Handbook of human-computer interaction. (Second Edition). Amsterdam: North-Holland. 733-766.

Metev SM, Veiko VP. 2006. Task structure and user attributes as elements of human-robot interaction design. Proceed- ings of IEEE ROMAN 06 Conference, Hatfield, United Kingdom.

Okuno HG, Nakadai K, Kitano H. 2002. Social interaction of humanoid robot based on audio-visual tracking. Proceedings of the International Conference on Industrial, Engineering \& Other Applications of Applied Intelligent Systems, IEA/AIE 2002, Cairns, Australia, June 17-20.

Preece J, Rogers Y, Sharp H. 2007. Interaction Design: Beyond Human Computer Interaction. John Wiley \& Sons. Access Date: 7/1/09.

Menegatti E. 2007. RoboCup Soccer Humanoid League Rules and Setup for the 2007 competition in Atlanta, USA. Available from: http://www.robocup.org. Access Date: 7/1/09.

Zhou C, Yue PK. 2004. Robo-Erectus: A low cost autonomous humanoid soccer robot. Adv Robotics, 18(7):717-720. 

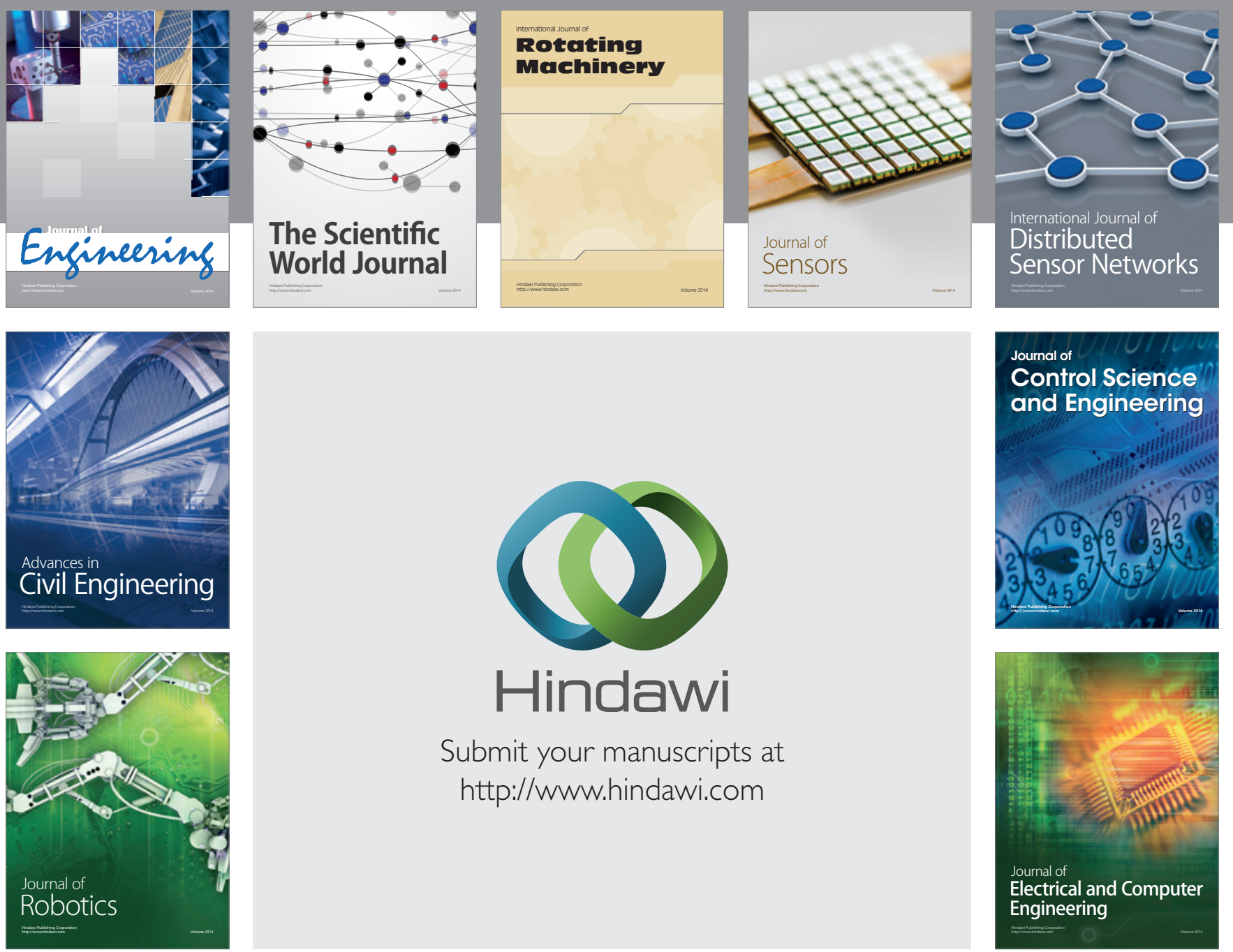

Submit your manuscripts at

http://www.hindawi.com
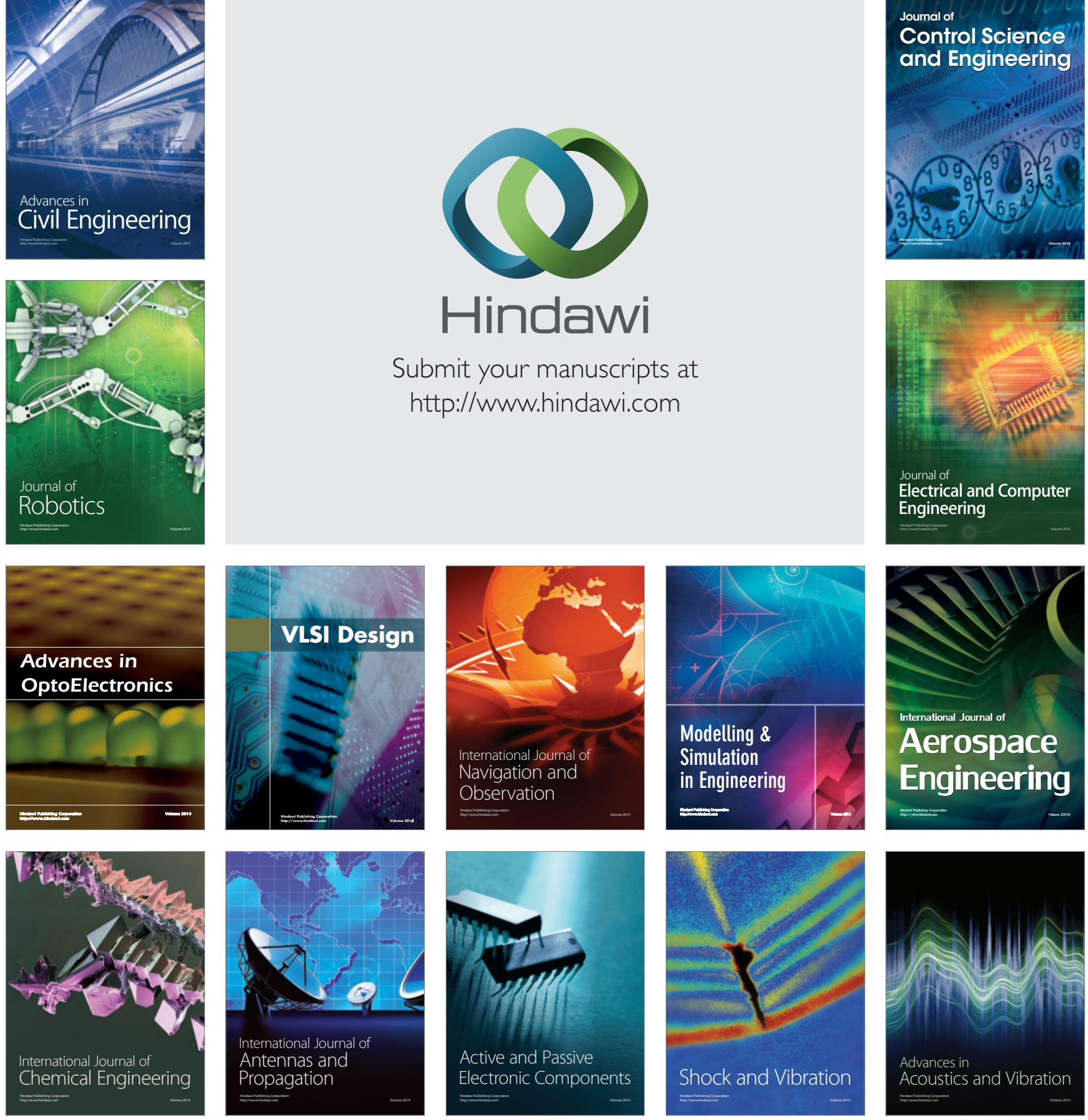